\title{
The Clinical Neuropathology of Spinal Cord Injury A Guide to the Future
}

\author{
Byron A. Kakulas A.O., M.D.(Hon.Athens), M.D.(W.A.), F.R.A.C.P., \\ F.R.C.Path. \\ Department of Neuropathology, Royal Perth Hospital and Neuromuscular Research \\ Institute, University of Western Australia
}

\section{Summary}

The neuropathology of spinal cord injury (SCI) is reviewed in the light of clinical problems and as a guide to future research. The pathology of SCI in the acute stage suggests that the spinal cord may be partly preserved even in the most severe injuries. This finding emphasises the need for great care in roadside management. In the acute phase there are irreversible changes and possibly reversible changes which have not been adequately identified. Even a small percentage of nerve fibres escaping the initial injury would be of great benefit to the patient.

In the subacute stage when transynaptic degeneration is proceeding there may also be associated functional changes leading to abnormal reflex activity. It is possible through an improved understanding of the neuropathology and neurophysiology of the isolated or partly isolated segments of the cord that new reflex connections may be stimulated to develop by artificial means. In the chronic stage there are well recognised complications such as osteoarthrosis with spinal stenosis, post-traumatic syringomyelia and traumatic nerve root neuroma formation, which may lead to clinical deterioration and which may be amenable to treatment.

In a more theoretical sense it is possible that improved understanding of CNS plasticity and transplant neurobiology using recombinant DNA technology, grafting and 're-education' of the regenerated tissues may be rewarding in the longterm future. Although this outcome is entirely hypothetical at this stage basic research deserves great emphasis.

Key words: Neuropathology of spinal injuries; Future research.

\section{Introduction}

Considerable knowledge of spinal cord injury (SCI) has accumulated as a result of detailed neuropathological evaluation of post mortem specimens over the past several years (Kakulas and Bedbrook, 1976; Kakulas, 1984). These studies were undertaken on patients who had died in the early, intermediate and late stages following a serious spinal injury. 
As a result the evolution and natural history of SCI and the corresponding clinical effects have become known with some accuracy. Up to the present time 225 traumatic cases among 341 spinal specimens with 41 normal controls have been studied and the discussion which follows derives from this experience. The pathology of the acute, subacute and chronic stages provides a framework on which to develop ideas for improved clinical procedures and future lines of research which may enhance the neurological status of the SCI patient.

\section{Neuropathological considerations}

\section{The acute stage}

A number of important conclusions may be drawn from a study of more than 100 severely traumatised spinal columns provided by the West Australian Coroner's Department. These subjects died at the roadside.

Among the findings is the observation that the spinal cord per se, despite its delicate semi-fluid texture, may fully or partially survive the initial injury even when damage to the bony structures, ligaments and muscles is extensive. In some of these cases the spinal cord parenchyma may even appear normal to the naked eye. In others the spinal cord is clearly damaged with petechial haemorrhages, oedema and disruption of parenchyma. In many of these specimens some continuity of long tracts seems to be preserved. Even when there are massive bony injuries, complete transection of the spinal cord is a rare finding.

However, some reserve is necessary in accepting the apparently intact cord on face value. The principles of pathology dictate that in many of these subjects it is very likely that an irreversible series of events was, in fact, initiated by the initial spinal cord injury, but that death occurred before they were set in train. That is to say, the initial damage on impact is probably very extensive at a submicroscopic level. Therefore if the patient had survived even for a few hours, necrosis would become visible. In a little time, if the patient had lived, this reaction would appear as 'traumatic inflammation', with myelomalacia and leucocytic infiltrates.

In other patients who do survive the initial injury, hyperaemia and other vascular changes become prominent in 12 to 24 hours. These reactions are mediated through prostaglandins, catecholamines and other agents. Thus necrosis manifests as haemorrhagic myelomalacia (Fig. 1). The vascular reaction subsides in a few weeks and gliosis ensues.

What is not known in the early stage is the degree of reversible or irreversible damage sustained in any one person. There are also complications to be kept in mind such as hypoxia and shock which may aggravate the initial lesion.

Therefore when considering possible improvements in the acute phase first among the immediate requirements is most careful roadside management. It seems that in many patients the spinal injury is aggravated by poor handling despite awareness of the problem and the introduction of careful transfer methods.

\section{The subacute stage}

One to 2 months post injury the neuropathology shows macrophage removal of debris and gliotic repair. Macrophages are actively engaged in ingesting necrotic 


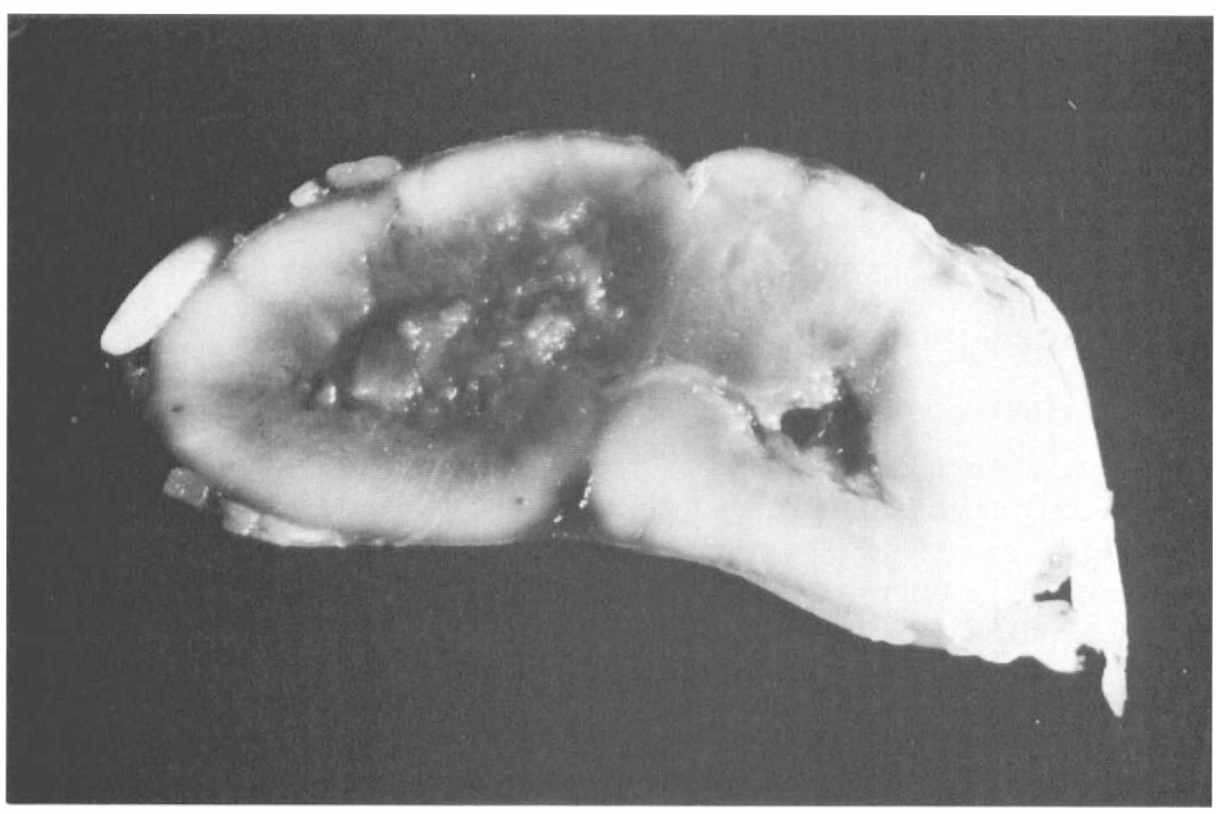

Figure Spinal cord in cross section at C5 showing central haemorrhagic necrosis and myelomalacia. Note the cavity formation and the preserved white matter at the periphery of the lesion. X69/141 Female of 20 yrs who suffered a C5 hyperextension injury and survived for only 10 days.

spinal cord tissue mainly in the central areas of the cord and the adjacent posterior columns, which suffer the brunt of the trauma. A glial network demarcates the central cavity from the white matter.

Perhaps the most important neuropathological change in the subacute stage is the continuing Wallerian degeneration and transynaptic changes occurring at points distal to the lesion. The interruption of the reticular system within the central grey matter may also be a contributing factor toward disordered spinal function. It may be inferred that concomitant changes might be occurring in the neuropeptides, chemical transmitters and hormones as a result of the injury but no factual information of this nature is available.

Disordered physiology might also contribute to a changing neurological status in the subacute period. It is well known that the central nervous system (CNS) requires a continuous sensory input to maintain its 'normal' integrity with associated synaptic and reflex activity. Thus reflex connections and patterns of activity established prior to the injury may become disorganised as a result of the disconnection from higher levels, exposure to abnormal sensory inputs, and disuse. 'Plasticity' is known to be an extremely dynamic process so that synapses and possibly dendritic arborisations may remodel and grow within the neural network, under suitable conditions.

The chronic stage

The neuropathology of the chronic stage is characterised by the presence of multilocular cysts with thick gliotic walls, nerve root regeneration and with a 
variable residuum of descending and ascending CNS fibres running in the walls of the lesions. The subarachnoid space is often obliterated by fibrous tissue which may also enter and mix with glial fibres. These meningoglial scars could be the source of abnormal neuropeptide release. Regenerating nerve roots, which are a feature of the chronic stage, rarely enlarge sufficiently to form a neuroma with compression of the cord remnant. It is possible, that the proximal axons of the posterior root ganglia when regenerated, may enter the posterior columns and find their way upward to connect with the nuclei cuneatus and gracilis.

There are complications in the chronic stage such as continuing osteoarthrosis of the bony column with resulting disc protrusions, osteophyte formation and increasing deformity. These changes may cause spinal stenosis and consequent neurological deterioration. Otherwise it is known that 3 to $5 \%$ of SCI patients may develop post traumatic syringomyelia (Barnett lesion). This complication may underlie a changing neurological picture with involvement of higher spinal cord segments. Another possible cause for deteriorating neurological function in the chronic phase, is vascular insufficiency. Atheromatous degeneration of the aorta and its branches which serve to augment the blood supply of the spinal cord may impair blood flow causing ischaemia of the spinal cord.

The surgical treatment of several of these conditions is straightforward. The relief of stenosis by decompression, joint fusion, and treatment of the cysts are examples.

In the context of the subacute and chronic lesions there are a number of therapeutic approaches which could theoretically, at least in the longterm future, be of benefit to the SCI patient. For instance, augmentation of existing descending pathways is a possible method of improvement. New neuronal connections with useful patterns of reflexes may possibly be induced via inputs which influence plasticity.

To be even more speculative and hypothetical the ultimate 'cure' of spinal paralysis by means of regeneration and transplantation (Kao et al., 1983) is a remote possibility worthy of consideration. Extensive knowledge of the molecular neurobiology of gene expression in the CNS is an essential prerequisite to this happy outcome. Knowledge of trophic factors, and surface molecules would provide the protein clues toward a molecular approach utilising the methods of recombinant DNA technology. Of course it will take years for useful information to accumulate but great advances may reasonably be expected in the future. If it were possible to restore growth and multiplication of nerve cells and their fibres and create new primary connections using auto-grafts (converted to primitive neuroblasts), 're-education' through the property of plasticity may also be a feasible proposition in the distant future.

\section{Conclusions}

It can be seen from this discussion that a knowledge of the neuropathology of spinal cord injury (SCI) is a useful starting point for consideration of new methods which might improve the neurological status of the SCI patient in the future.

Following a brief review of these neuropathological principles an attempt is 
made to suggest future lines of research which might ultimately solve the SCI problem. In brief these avenues consist of, improved roadside management, modification or augmentation of residual neurological functions in established injuries and the eventual reconstruction of the injured spinal cord as a result of advances in molecular neurobiology.

\section{References}

KaKulas BA, Bedbrook GM 1976 Injuries of the spine and spinal cord. In: VinKen PJ, BrUYN GW (eds) Handbook of Clinical Neurology, Chapter 3. North Holland Publishing Company, Amsterdam, pp. 27-42

KaKulas BA 1984 Pathology of spinal injuries. Central Nervous System Trauma 1:117-129

Kao CC, Bunge RP, ReIER PJ (eds) 1983 Spinal Cord Reconstruction, Raven Press 